\title{
Analytical chemistry, multidimensional spectral signatures, and the future of coherent multidimensional spectroscopy
}

John C. Wright

Department of Chemistry, University of Wisconsin, Madison, WI 53706 United States

Email address: wright@chem.wisc.edu

\begin{abstract}
Spectroscopy is a dominant measurement methodology because it resolves molecular level details over a wide concentration range. Its limitations, however, become challenged when applied to complex materials. Coherent multidimensional spectroscopy (CMDS) is the optical analogue of multidimensional NMR and like NMR, its multidimensionality promises to increase the spectral selectivity of vibrational and electronic spectroscopy. This article explores whether this promise can make CMDS a dominant spectroscopic method throughout the sciences. In order for CMDS to become a dominant methodology, it must create multidimensional spectral fingerprints that provide the selectivity required for probing complex samples. Pump-CMDS probe methods separate the pump's measurement of dynamics from a multidimensional and selective probe. Fully coherent CMDS methods are ideal multidimensional probes because they avoid relaxation effects, spectrally isolate the output signals, and provide unique and invariant spectral signatures using any combination of vibrational and electronic quantum states.
\end{abstract}

\section{Introduction}

Spectroscopy is an important analytical method for almost every area of science because of its ability to discriminate between the different components in materials over a wide range of concentrations. Vibrational spectra are particularly selective because their numerous, narrow features act as spectral signatures of specific components and moieties. Spectral signatures in this paper are defined either as a single peak or a pattern of peaks that uniquely characterize a single component. Multidimensional NMR methods like heteronuclear multiple quantum coherence (HMQC) have even higher selectivity. ${ }^{1}$ These are fully coherent methods that resolve the nuclear spins within specific residues of complex proteins containing thousands of atoms. ${ }^{2,3}$ They form the basis for NMR's ability to measure protein structure. ${ }^{4}$ The high selectivity results from the formation of multiple quantum coherences (MQCs) between nuclei like ${ }^{1} \mathrm{H},{ }^{13} \mathrm{C}$, and ${ }^{15} \mathrm{~N}$. Crosspeaks appear in the multidimensional spectra if the spins are coupled. The chemical shifts of each spin create cross-peaks that act as spectral signatures of specific residues in complex proteins.

A new family of coherent optical spectroscopies were first described in 1997. ${ }^{5}$ They were based on the analogue of NMR MQCs. Instead of nuclear spin states, the MQCs in this proposed new family use a mixture of vibrational states to create spectral signatures of materials. MQCs form the basis for the field of Coherent Multidimensional Spectroscopy (CMDS). ${ }^{6,7}$ CMDS is proving to be a powerful tool for measuring ultrafast dynamics in a wide variety of systems and it promises to be a transformative technology for revolutionizing the spectroscopic discrimination required for wide-spread dissemination. ${ }^{8}$ The future of CMDS depends on two factors that are central to wide-spread dissemination: the ability of CMDS methods to create 
multidimensional spectral signatures that can address important measurement challenges and the development of lower cost and easily used instrumentation.

\section{Background}

CMDS uses different excitation frequencies to rapidly excite electronic and vibrational states to form a MQC, i.e. a quantum mechanical superposition state described by a wave function, $\Psi(x, t)=\sum_{n} c_{n} \psi_{n}(x) e^{i\left(\vec{k}_{n} \llbracket-\omega_{n} t\right)}$, that defines its amplitude, spatial shape, and temporal and spatial oscillations. ${ }^{9-14}$ States within the MQC can be created by single transitions from the ground state or by sequential transitions involving other states. The states involved in sequential transitions inherit the spatial and temporal oscillations of the earlier states and excitation fields. We concentrate first on four wave mixing (FWM) CMDS where three excitation pulses create a fourth output pulse. As an example, consider three excitation pulses tuned to the $g \rightarrow a, g \rightarrow b$, and $b \rightarrow c$ transitions where $g$ is the ground state and $a, b$, and $c$ are vibrational or electronic states. $^{15,16}$ Together they create a MQC involving states $g, a, b$, and $c$. If there are interactions between these states, i.e. the states are coupled, the MQC will provide a three-dimensional spectral signature that brings the full power of coherent methods to analytical measurements. ${ }^{17}$

Each pair of states such as $c$ and $a$ represents a coherence that oscillates spatially and temporally, $\psi_{c} \psi_{a}^{*} e^{i\left(\vec{k}_{c a} \llbracket-\omega_{c a} t\right)}$. If the oscillating $c a$ coherence formed by three field interactions has a transition dipole moment, $\vec{\mu}_{c a}$, then the ensemble of oscillating coherences forms a spatially and temporally modulated nonlinear polarization that launches an electromagnetic field at the frequency difference, $\omega_{c a}$. The third order nonlinear susceptibility, ${ }_{\chi}^{(3)}$, is a fourth rank tensor relating the polarization components of the nonlinear polarization and the electromagnetic fields of the excitation pulses, $\vec{P}==^{=(3)} \sum_{i} \vec{E}_{i}$. If the wave-vector of the electromagnetic wave, $\vec{k}_{\text {out }}$, matches the wave-vector of the polarization that launched the wave, $\vec{k}_{c a}$, the field created by the ensemble of coherences within the excitation volume will constructively interfere to form a bright, directional beam whose intensity depends quadratically on the concentration of oscillators and the excitation path length. The emission is driven by the fields during the excitation stage and continues after as free induction decay (FID). The beam will exist until the collective coherences within the excitation volume lose their relative phases because the chaotic environment randomizes the coherence frequencies. The emission then continues as incoherent fluorescence whose intensity scales linearly with concentration. Fluorescence continues until the excited state population decays. It should also be noted that all pairs of states within the MQC simultaneously create their own beams that may or may not be phase matched. For example, the $c g$ coherence within the MQC is formed by only two sequential interactions, $g \rightarrow b \rightarrow c$. It creates a nonlinear polarization that can launch an electromagnetic field. It is described by a second order susceptibility, $\chi^{(2)}$, and forms the basis for interface sensitive methods such as sum frequency generation (SFG). ${ }^{18,19}$ 
There are two time ordered sequences of transitions that can form the $c a$ coherence, $g g \rightarrow g a \rightarrow b a \rightarrow c a$ and $g g \rightarrow b g \rightarrow b a \rightarrow c a$ where the labels represent the two states of the corresponding density matrices, $\rho_{m n} \equiv c_{m} c_{n}^{*}$. The pathways are fully coherent because there are no intermediate populations. If the excitation pulses are long compared to the dephasing times of the $g a, b g$, and $b a$ coherences, the two pathways contribute equally and add. Their relative contributions affect the line shape of spectral features. If the excitation pulses are shorter than the dephasing times, the pathways can be isolated by time ordering the excitation pulses. If the $a$ and $b$ states are identical and both excite state $b$, the $b a$ coherence becomes $b b$ which is the population of the $b$ state. It does not oscillate temporally. Such population pathways are partially coherent and the spectral signature becomes two-dimensional.

The resonances of the two pathways can be visualized by the Wave-Mixing Energy Level (WMEL) ${ }^{20}$ diagrams in Fig. 1 where the solid lines follow the sequence of transitions leading to the upper state (c) of the output coherence and the dotted lines follow the sequence leading to the bottom state (a). The first two diagrams differ only in their time ordering. The time ordering proceeds from left to right where the last arrow is the output transition created by the $c a$ coherence. The magnitude of the output field coherence is proportional to the amplitude of $\rho_{c a}$. In the steady state, $\rho_{c a}$ depends on the excitation field, the transition dipole moments $(\mu)$, the detuning from resonance $\left(\omega_{m n}-\omega_{i}\right)$, and the dephasing rate $\left(\Gamma_{m n}\right)$ $\rho_{c a}=-\frac{\left(\vec{\mu}_{g a} \cdot \vec{E}_{1}\right)\left(\vec{\mu}_{g b} \cdot \vec{E}_{2}\right)\left(\vec{\mu}_{b a} \cdot \vec{E}_{3}\right) e^{i\left[\left(-\vec{k}_{1}+\vec{k}_{2}+\vec{k}_{3}\right) \cdot \vec{z}-\left(-\omega_{1}+\omega_{2}+\omega_{3}\right) t\right]}}{8 \hbar^{3}\left(\omega_{a g}-\omega_{1}+i \Gamma_{a g}\right)\left(\omega_{b a}-\omega_{1}+\omega_{2}-i \Gamma_{b a}\right)\left(\omega_{c a}-\omega_{1}+\omega_{2}-\omega_{3}-i \Gamma_{c a}\right)} \cdot{ }^{10}$ The exponential defines the spatial and temporal modulations of the $c a$ coherence created by the wave vectors and frequencies of the three excitation fields. The output is multiplicatively enhanced by each of the three resonance denominators. At resonance, the coherence created by each successive interaction scales as the ratio of the Rabi frequency $\left(\frac{\vec{\mu}_{g a} \cdot \vec{E}_{1}}{\hbar}\right)$ to the dephasing rate. The $\rho_{c a}$ coherence is proportional to the dipole moment that launches the output field. The ensemble of oscillating dipoles constitutes a nonlinear polarization whose direction is defined by $-\vec{k}_{1}+\vec{k}_{2}+\vec{k}_{3}$. The ensemble's emission can constructively interfere and create cooperative 
emission if the k-vector of the electromagnetic field matches the polarization wave vector, $\vec{k}_{\text {out }}=-\vec{k}_{1}+\vec{k}_{2}+\vec{k}_{3}$, i.e. phase matching. The last diagram shows a partially coherent pathway where the first two interactions create a static population in state $b$.

CMDS experiments are performed in the frequency and time domain. ${ }^{21}$ In the frequency domain, the pulses are longer than the dephasing times and emission occurs dominantly by the excitation pulses driving the output beam. The long excitation pulses have a narrow spectral bandwidth that resolves individual quantum states. ${ }^{10}$ Scanning the excitation frequencies across the three resonances of $\rho_{c a}$ results in resonance enhancements of the output signal. The three resonances multiplicatively enhance the output intensity that can result in 3D spectra. In the time domain, the pulses are shorter than the dephasing times so scanning the delay times between excitation pulses resolves the FID phase oscillations of the coherences. ${ }^{22-25}$ These phase oscillations are visualized in the bottom of Figure 1. Actually measuring the phase oscillations requires a phase reference that is provided by a local oscillator that has a similar frequency to the output beam. The output signal and local oscillator electric fields are spatially overlapped so they heterodyne and create the fringes that define the phase oscillations of each coherence as the delay times change. Fourier transform of the phase oscillations then creates multidimensional spectra. Time domain methods require phase stability between the output and local oscillator fields within $\sim \lambda / 100$ in order to avoid artifacts. ${ }^{26}$ The phase stability is usually attained by splitting a beam into the three excitation beams required for a CMDS experiment and a fourth beam that acts as the local oscillator. The states forming an MQC are then limited to those that can be excited by the bandwidth of the ultrafast pulses, typically $140-2,700 \mathrm{~cm}^{-1} \cdot{ }^{27,28}$ Two separate beams with different colors can also be used with a partially coherent pathway because the phase oscillations of the output coherence are isolated from the phase oscillations of the first coherence by the static intermediate population. ${ }^{29}$

CMDS can also be done in a mixed frequency/time domain where the excitation pulse widths are comparable to the dephasing time of the coherences. ${ }^{7}$ The spectral band width is then narrow enough to excite individual quantum states while the temporal width is short enough to resolve the dynamics. The output signals in frequency domain methods depend only on having coherent excitation pulses to impart the spatial and temporal phase relationships that create the output beam. Long term phase coherence is not required between the output and local oscillator fields as it is in time domain methods. Multiple excitation frequencies can therefore create an MQC containing any combination of vibrational and electronic states.

\section{Current Capabilities of CMDS- Photon Echo, Pump-Probe, and Transient Absorption}

\subsection{Inhomogeneous Broadening and Line Narrowing}

One of the most important capabilities of CMDS methods is resolving spectral features buried within inhomogeneously broadened transitions. Inhomogeneous broadening occurs when the frequencies of the molecular vibrational and electronic states are shifted by their different environments and conformations. In time domain methods, the ensemble of frequencies leads to a more rapid loss of phase coherence during the FID. In the frequency domain, the ensemble of frequencies leads to an inhomogeneously broadened Gaussian peak. CMDS methods can narrow 
inhomogeneously broadened peaks. In the frequency domain, the narrowing occurs when the excitation frequencies are multiply resonant with a specific subset of states within the ensemble. The multiply resonant transitions contribute a dominant multiplicative enhancement at the fully resonant frequencies of the subset. Other states within the ensemble have fewer resonances and smaller net enhancements. In the time domain, line narrowing occurs because of a rephasing of the coherences.

The inhomogeneous environments can change on time scales ranging from years to femtoseconds. Ultrafast time scales result in the dephasing rates that determine the width of homogeneously broadened transitions. At longer times, inhomogeneous broadening also causes dephasing in the ensemble but it can be reversed. Pathways like $g g \rightarrow g a \rightarrow a a \rightarrow a g$ involve the $g a$ initial coherence and conjugate $a g$ output coherences whose phase evolves in time as $e^{i\left(\omega_{a g}^{o}+\delta \omega\right) t}$ and $e^{-i\left(\omega_{a_{g}}^{o}+\delta \omega\right) t}$, respectively, where $\delta \omega$ is the frequency shift induced by the random environment. ${ }^{12,13,30-32}$ The signal created by the ag output coherence would rapidly vanish because the inhomogeneous distribution of $\delta \omega$ values would destroy the phase coherence of the ensemble. However, the signal would reappear when the delay time matched that of the original $g a$ coherence since the phase differences vanish at that point. This pathway is an example of a photon echo. The size of the echo depends upon the lifetime of state a, the dephasing rate of the $g a$ and $a g$ coherences, and any randomization that occurs in the $\omega_{a g}^{o}$ frequency over delay times when populations exist. ${ }^{33}$ The rephasing and associated echo only enhances the signal for molecules when the $\delta \omega$ values for the initial $g a$ and final $a g$ coherences are identical.

Fig. 2 shows a series of 2D spectra of the amide stretch region obtained with the photon echo pathway at different times in the formation of amyloid fibers in a human islet amyloid polypeptide. ${ }^{34}$ The $\mathrm{x}$ - and $\mathrm{y}$-axes define the excitation frequencies of the $a g$ and $g a$ coherences, respectively. The spectra have a strong diagonal character because the initial and final frequencies are correlated since they both arise from molecules in the same environment. There are two features characterized by the red and blue color scales. The red feature results from pathways creating the $a g$ output coherence while the blue feature results from the excited state absorption pathway involving the overtone of the amide stretch, $g g \rightarrow g a \rightarrow a a \rightarrow 2 a, a$. The colors
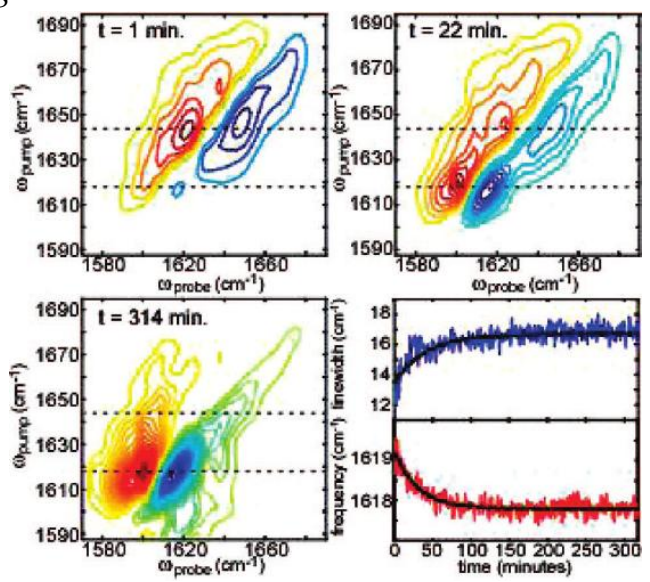

Figure 2-Figure 2-2D-IR spectra of an amyloid peptide at different times in the formation of amyloid fibers. reflect the $180^{\circ}$ phase difference of the $a g$ and $2 a, a$ output coherences relative to the local oscillator. The diagonal width reflects the inhomogeneous broadening and the antidiagonal width reflects the homogeneous broadening. Homogeneous broadening probes the ultrafast fluctuations of the environment that surrounds a molecule. By attaching different ${ }^{13} \mathrm{C}-{ }^{18} \mathrm{O}$ isotopic probes into different residues along the length of the polypeptide transmembrane helical bundle and measuring the differences in the homogeneous and inhomogeneous broadening, the Zanni group showed that the terminal residues of the 
polypeptide that were near the membrane surface were more inhomogeneously broadened while the residues near the middle were more homogeneously broadened. ${ }^{27}$

\subsection{Spectral Diffusion}

The strong diagonal character in Fig. 2 is characteristic of static inhomogeneous broadening. If spectral diffusion occurs because the environment is not static and the frequencies of the coherences change during the time the system is in a static population, the rephasing will become less important and the diagonal character disappears. Fig. 3 shows an example of spectral diffusion on a functionalized alkanethiol self-

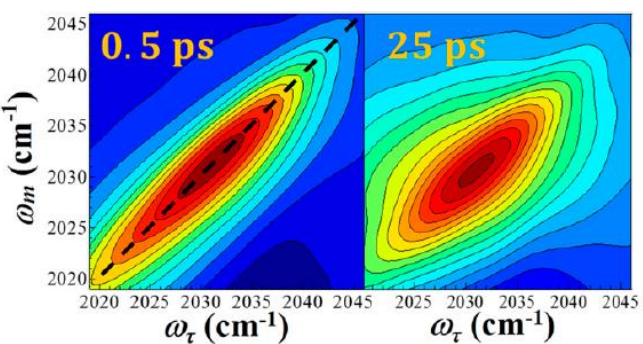

Figure 3-2D-IR spectra of a vibrational probe molecule in a self-assembled monolayer on gold for two different population times. The dashed lines define the diagonal. assembled monolayer on gold using a photon echo pathway and a metal carbonyl vibrational probe at the head group. ${ }^{35}$ At early times, the carbonyl mode is strongly diagonal with a center line slope (CLS) of 1. After the vibrational population has existed for $25 \mathrm{ps}$, the CLS has decreased to 0.76. The CLS is a measure of the frequencyfrequency correlation function between the initial frequency and later times. ${ }^{36,37}$

\subsection{Chemical Exchange Dynamics}

CMDS is also sensitive to chemical reactions where the excited molecule changes. Fig. 4 shows an example of a reaction between phenol and benzene. ${ }^{36}$ In the upper figure, the population time between the creation of the initial and final coherence is short so two diagonal peaks appear. They correspond to free phenol molecules and phenol-benzene complexes. After a 14 ps delay, cross-peaks appear between the two features. The first two interactions excite a population in one of the two species. During the 14 ps delay, the species change, either by dissociation of the complex (upper left peak) or the formation of a complex with the benzene (lower right peak). The strength of the cross-peak measures the reaction rate forming or dissociating the complex.

\subsection{Vibrational Energy Transfer and Relaxation Assisted Spectroscopy}

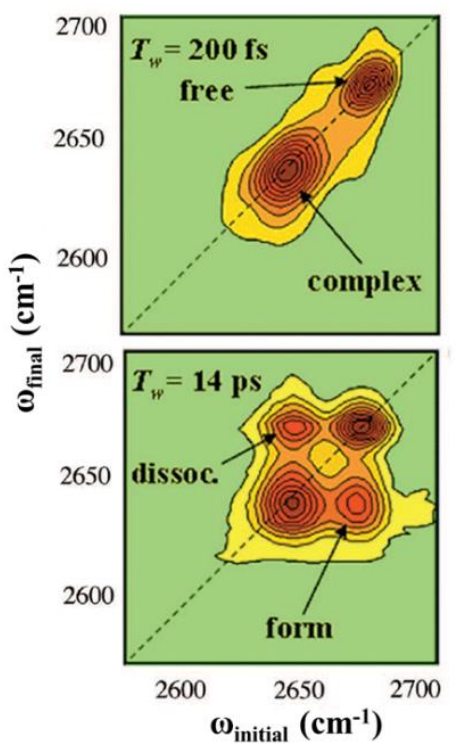

Figure 4-2D-IR spectra of a phenolbenzene mixture for $200 \mathrm{fs}$ and $14 \mathrm{ps}$ population delay times. The free phenol vibrational mode appears at $2665 \mathrm{~cm}-1$. It shifts to $2631 \mathrm{~cm}-1$ when the phenol forms a complex with benzene.

Population transfer between vibrational states also occurs during the second time delay. Excitation of a particular vibrational mode results in a new cross-peak whose intensity reflects the rate of transfer to the new mode. Cross-peaks can be significantly enhanced by exciting a mode with a large transition moment and allowing vibrational relaxation to populate another mode with a lower transition moment. In addition, this 
relaxation assisted spectroscopy detects the bond connectivity patterns by following the relative rise-time dynamics of the different cross-peaks. Since the vibrational energy transfer rate depends strongly on distance, it provides information on molecular dimensions and mode delocalization. $^{38}$

\subsection{Structure Determination}

CMDS methods can also use polarized excitation and output beams to directly probe the elements of the nonlinear susceptibility tensor and provide a powerful way to probe the symmetry of a molecule. Fig. 5 shows a series of absolute magnitude $2 \mathrm{D}$ spectra of a model Nacetyl-L-prolinamide dipeptide. ${ }^{39}$ The columns show spectra taken with phase matching conditions for the photon echo rephasing pathway, the non-rephasing pathway, and the reverse photon echo pathway, respectively. These pathways differ in the phase matching and pulse time

$(0,0,0,0)$

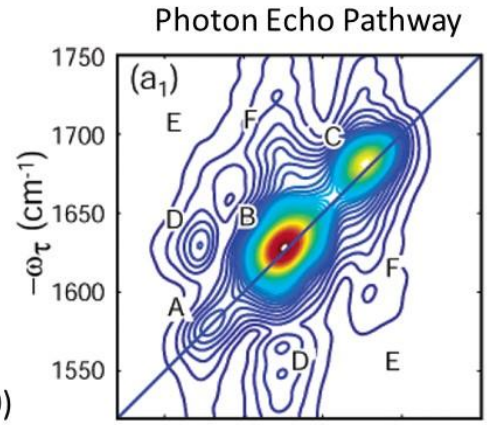

Polarizations
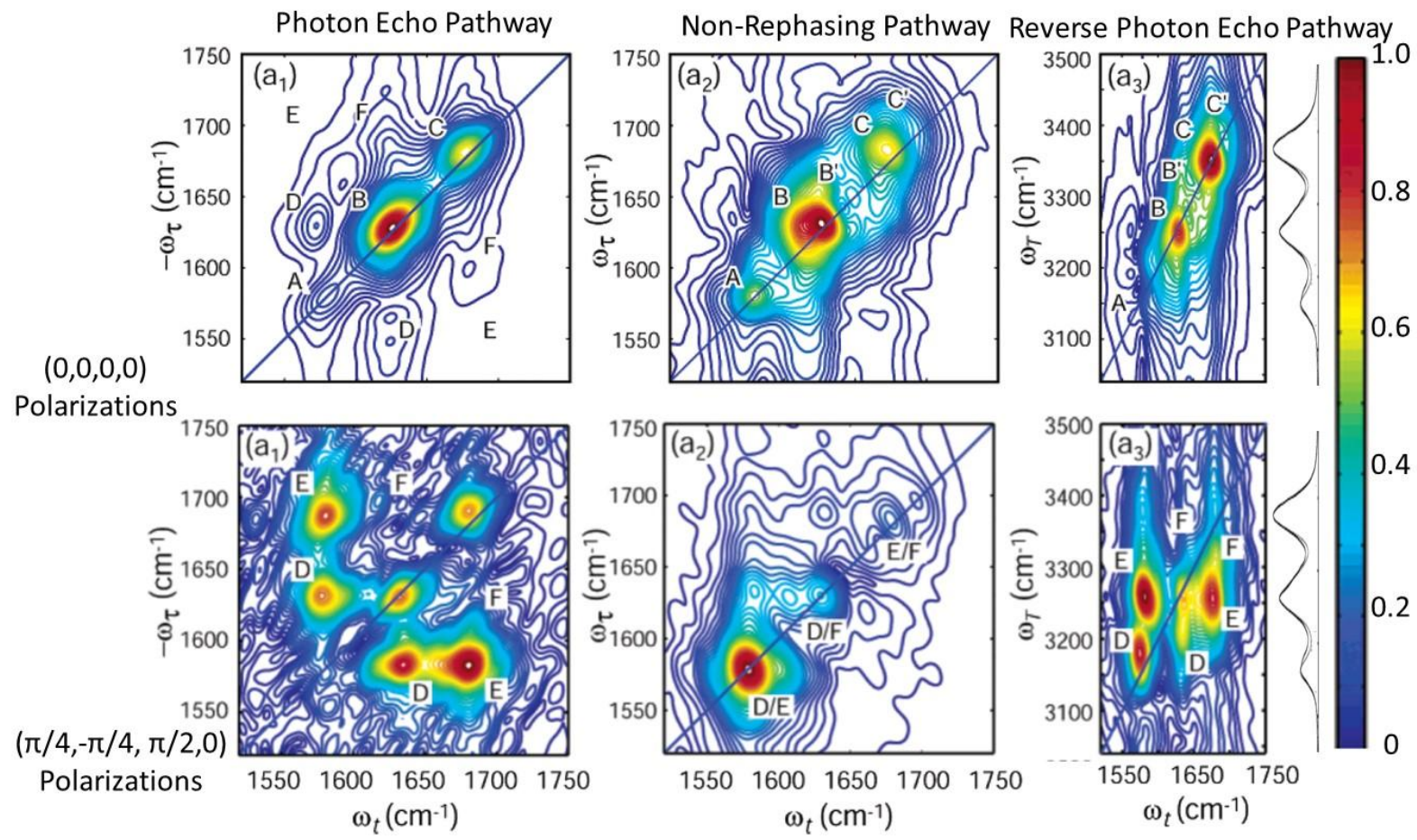

Figure 5-2D-IR spectra of an octapeptide using pathways with three phase matching conditions and two different sets of polarizations.

ordering. The rows show spectra with two different polarization conditions for the three excitation pulses and the output beam. The $(\pi / 4,-\pi / 4, \pi / 2,0)$ crossed polarization condition in the rephasing pathway suppresses diagonal peaks so the cross-peaks appear more clearly. The AF letters label the different spectral features that appear in the different pathways and polarization conditions. The infrared absorption spectra are shown on the right insert. The increase in spectral detail provided by the multiple dimensions, polarizations, and pathways is striking. Simulation of these spectra provided the angles between transition dipoles, coupling constants, and offdiagonal anharmonicities of the amide modes. The simulations matched the experimental data and showed that the dipeptide exists in multiple conformers of this dipeptide. This experiment is 
an excellent example of how the use of polarized light and multiple pathways provide the necessary information for structural determinations.

\subsection{Dual frequency CMDS}

The experiments described up to this point were restricted to exciting quantum states over the spectral bandwidth of ultrafast pulses. The limitation results from the need to maintain stable longtime phase coherence between excitation pulses while acquiring time domain data. Excitation pulses with different frequencies do not usually have the long time phase relationships required to perform fully coherent experiments. This restriction is removed by dual frequency experiments that use partially coherent pathways. These experiments have excited state populations with a well-defined static spatial modulation that defines

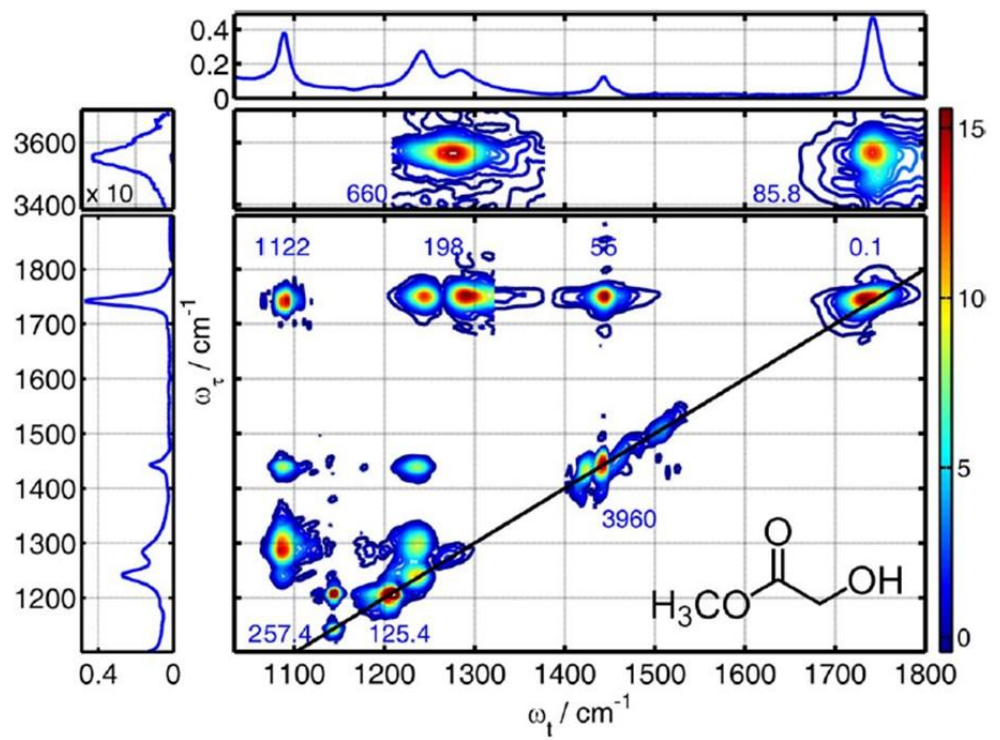

Figure 6-2D-IR spectrum of methylglycolate with each region of peaks normalized individually. The numbers denote the relative magnifications. The side plots show the absorption spectra. first two interactions. The phases of the initial and final coherences are isolated from each other by the static population. It is then possible to extend the capabilities over the wide range of frequencies characteristic of most spectroscopic investigations. A variety of different experimental approaches have successfully adopted dual frequency CMDS. ${ }^{40-42}$ Figure 6 shows an example dual frequency spectrum of methylglycolate that covers the entire fingerprint region of the infrared. ${ }^{43}$ It was formed by combining a series of narrow region spectra. The dynamic range in this spectrum is $>10^{5}$ and is normalized by the magnification to the brightest feature in each cluster of peaks. The brightest peak is demagnified by 0.1 and the dimmest is magnified by 3960 . The linear absorption spectrum is displayed on the side and top. Obtaining reproducible relative intensities is difficult because the alignment and phase matching of the three excitation beams and local oscillator change over the frequency range.

\subsection{CMDS Methods with Incoherent Detection}

The CMDS methods to this point are based on four wave mixing and the creation of coherent output beams. It is also possible to add an additional interaction that creates a static excited state population that can fluoresce or change other properties of a material such as a photocurrent or photovoltage. ${ }^{44-46}$ An example of an incoherent detected photon echo pathway is $g g \rightarrow g a \rightarrow g g \rightarrow b g \rightarrow b b$ where the $b b$ state population fluoresces or generates a photocurrent. In order to define the frequencies of the ga and bg coherences, the phase of each excitation beam is modulated at separate frequencies while monitoring the strength of the 
incoherent output signal. The ouput signal has many modulation frequencies that reflect the number of interactions with each excitation beam. Phase sensitive detection of the desired output modulation frequencies then resolves each contribution of the individual pathways.

4. A New Family of Fully Coherent Spectroscopies

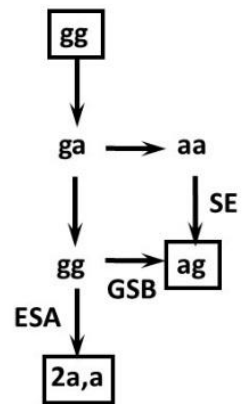


Partially coherent CMDS methods like photon echo, pump-probe, and transient absorption spectroscopies provide a powerful way to follow population dynamics on ultrafast time scales. ${ }^{13,47-50}$ Each method is described by three similar coherence pathways. For photon echo, these pathways are $g g \rightarrow g a \rightarrow g g \rightarrow a g, g g \rightarrow g a \rightarrow a a \rightarrow a g$, and $g g \rightarrow g a \rightarrow a a \rightarrow 2 a, a$. They correspond to ground state bleaching (GSB), stimulated emission (SE), and excited state absorption (ESA). They all occur simultaneously and they interfere. Figure 7 shows their relationships. The boxes identify the $g g$ initial ground state population and the $a g$ and $2 a, a$ output coherences. The ESA pathway has the opposite sign from the other pathways and it destructively interferes. If coupling is absent, the interference is complete and no peaks appear. If mechanical or electrical anharmonicity creates coupling between states, the interference is incomplete and diagonal and/or cross-peaks appear. The coupling can change the transition moments, the coherence frequencies, and the dephasing rates. ${ }^{51-56}$ Electrical anharmonicity between two vibrational modes changes the transition moments. Mechanical anharmonicity changes

Figure 7-The GSB, SE, and ESA pathways for the photon pathways of a vibrational state, $a$, and its overtone state, 2a. The boxes denote the initial gg ground state population and the ag and $2 a$, a output coherences. the coherence frequencies, and the interaction with the environment changes the dephasing rates. Finally, it is important to realize that the output signal frequency from partially coherent pathways is the same as an excitation frequency so spectral discrimination between the output signal and scattering from the excitation beams is not possible.

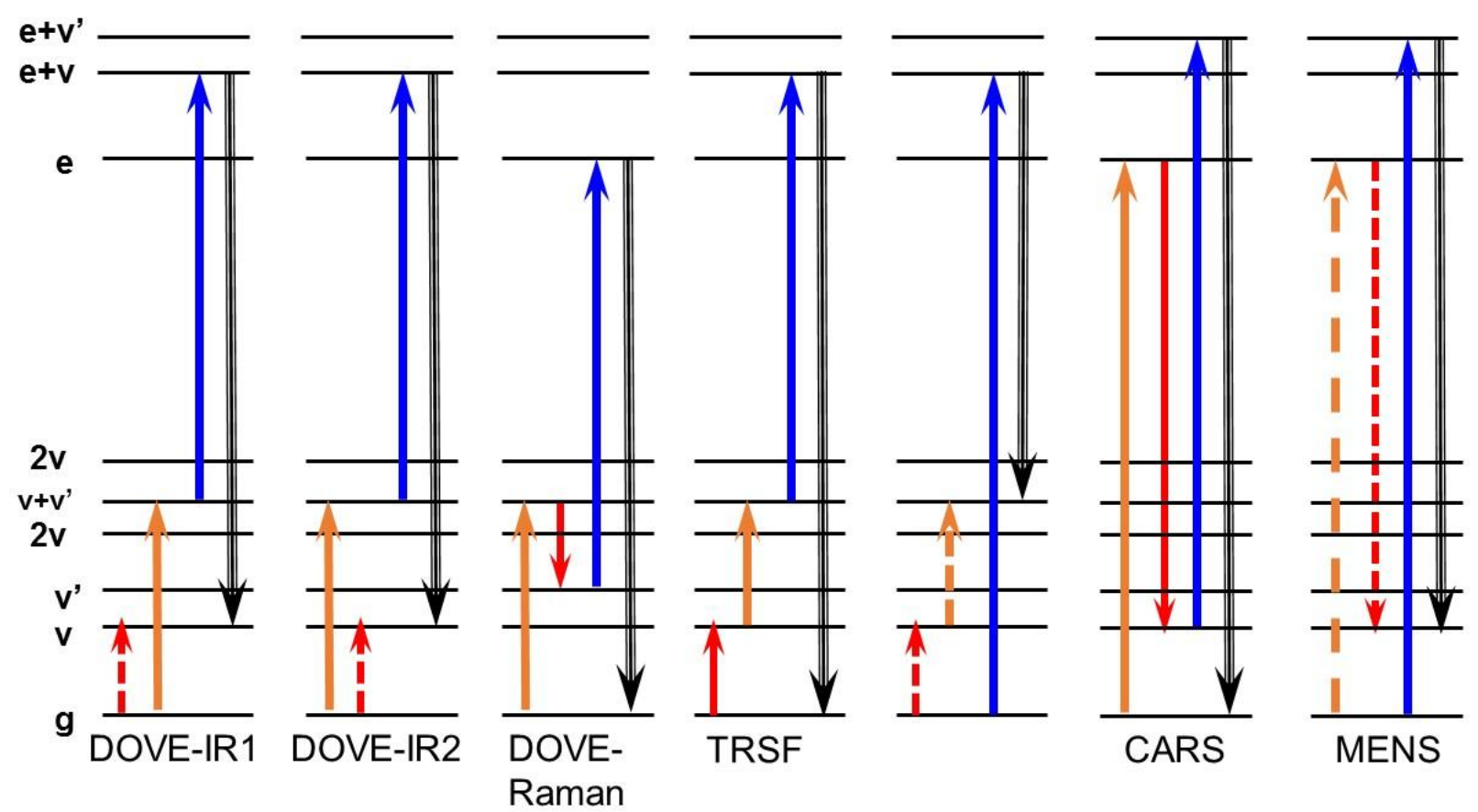

Figure 8-Fully coherent pathways involving $v$ and $v^{\prime}$ fundamental, overtone, and combination band states and e, e+v, and e+v' excited electronic and vibronic states. The interactions leading to the states involving in the output coherences appear as solid and dotted arrows

Fully coherent pathways are complementary to the partially coherent pathways. ${ }^{9,57}$ If the three excitation frequencies are different, the output frequency is different as well. The output frequency can be spectrally resolved from the excitation frequencies and eliminate scattering interference. These pathways lack intermediate populations so population relaxation and spectral diffusion effects are also absent from the spectra. The cross-peaks in fully coherent 
multidimensional spectra reflect the direct coupling between the states in MQCs and serve as excellent multidimensional spectral signatures. ${ }^{8,17,58}$ The original proposal for a CMDS family of fully coherent methods was based on the idea of using the resonance enhancements of two vibrational transitions to provide high selectivity and multidimensional spectral signatures and an electronic resonance to provide large resonance enhancements and additional selectivity. ${ }^{5}$ The WMEL diagrams for these methods appear in Figure 7. The first 4 and the last 2 have been experimentally realized. ${ }^{15,16,59-75}$ The pathways differ in the time ordering of the excitation pulses, the sequence of resonances, the output frequency, and whether the pathways are parametric or non-parametric, i.e. whether or not the molecular system returns to its original state. DOVE-Raman, TRSF, and CARS are parametric processes. The others are non-parametric. 
The earliest examples of CMDS methods used the triply resonant coherent Raman methods named coherent antiStokes Raman Spectroscopy (CARS) and Multiply Enhanced Nonparametric Spectroscopy (MENS). The pathways are shown in Figure 8. ${ }^{10,67-74,76-80}$ Both involve resonances with an electronic state, a vibrational state, and a vibronic state. MENS differs because its first two coherences are phase conjugates so the MENS pathway is a rephasing and line-narrowing pathway. Figure 9 shows example spectra of the fully resonant CARS of pentacene doped benzoic acid crystals at $2 \mathrm{~K} .{ }^{69,81}$ Under these conditions, the absorption and fluorescence spectra have 30 and 29 transitions, respectively. The fluorescence spectrum defines the ground state vibrational frequencies and the absorption spectrum defines the excited electronic state
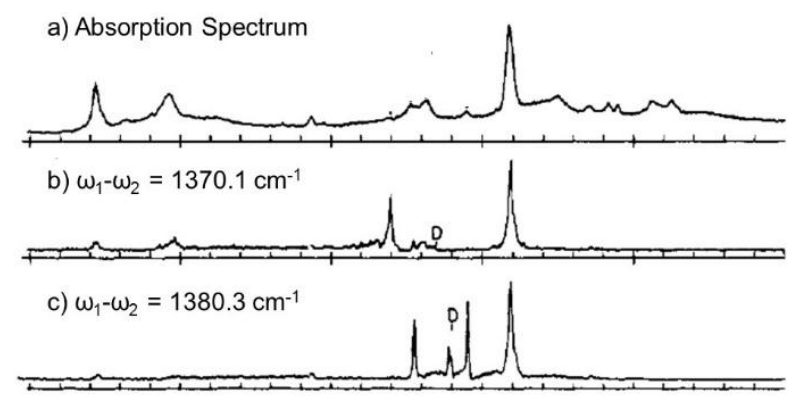

d) $\omega_{1}-\omega_{2}=1384.1 \mathrm{~cm}^{-1} \quad$ ।
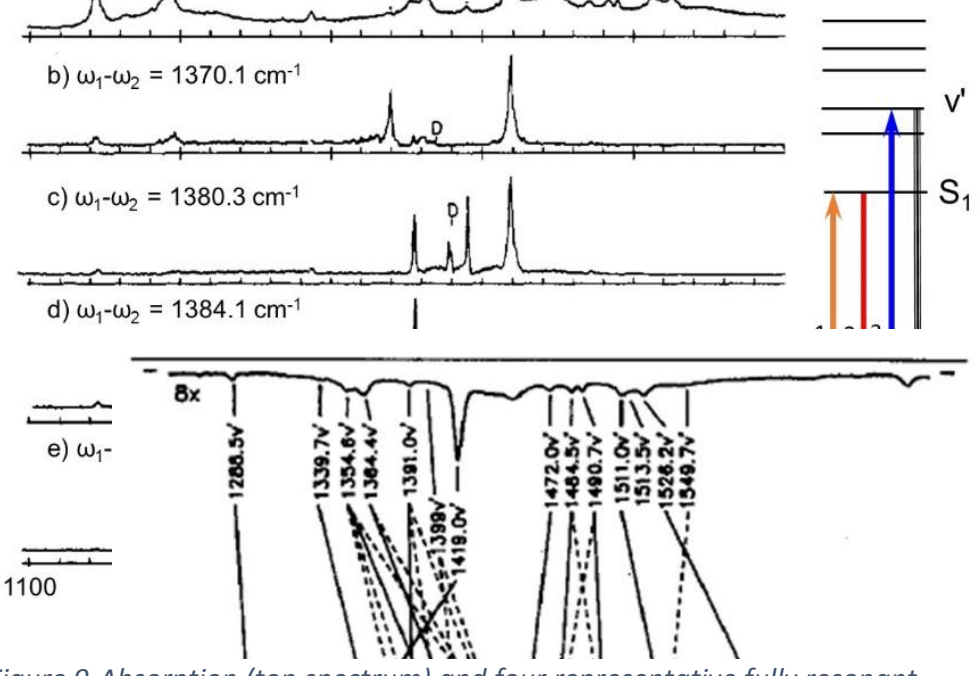
Figure 9-Absorption (top spectrum) and four representative fully resonant CARS spectra of pentacene in a benzoic acid crystal at $2 \mathrm{~K}$. The first excitation is resonant with the S1 electronic transition. The second transition is resonant with the indicated vibrational mode. The third transition is scanned across the different vibronic modes. The position where $\omega 1=\omega 3$ is indicated by $a$ D.

vibronic frequencies. The features have line widths ranging from $2-7 \mathrm{~cm}^{-1}$

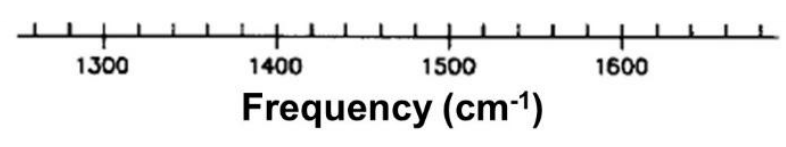

Figure 10-Correlation map between the frequencies of the vibronic states in the absorption spectrum (top) and the vibrational states in the fluorescence spectra (bottom).

\section{$\mathrm{S}_{1}$ zero phonon electronic state (FWHM of}

$\left.2.1 \mathrm{~cm}^{-1}\right)$. The second resonance is established with each of the 29 vibrational states observed in the fluorescence spectrum (only four are shown). The third resonance is scanned across the 30 vibronic states (labeled v') in the $S_{1}$ state. The series of spectra correlate all the vibrational and vibronic states observed in the absorption and fluorescence spectra. The absorption spectrum is displayed at the top as a comparison with the CARS spectra. The change in the molecular geometry between the $S_{0}$ and $S_{1}$ states mixes the modes and the correlations define the mixing and allow a determination of the excited state structure. Figure 10 summarizes the an example of the correlations between the vibrational and vibronic features. ${ }^{68}$ The lines correlate the vibronic features in the absorption spectrum at the top with their vibrational features in the fluorescence spectrum at the bottom. Solid lines denote the strongest correlations and the dotted lines show the weaker correlations. The correlations depend on the mixing of modes that is caused by the geometry change that pentacene undergoes in the excited electronic state.

CARS is not a rephasing pathway and does not allow line-narrowing of the inhomogeneous broadening. The MENS pathways use the same resonance enhancements but the coherences are different. In particular, the coherences in the MENS pathways have the same relationships as those in photon echo pathways. They contain two conjugate coherences with the oppositely signed temporal oscillations that are required for line-narrowing of inhomogeneous broadening ${ }^{70-73,78,79}$ Together, CARS and MENS provide the CMDS family of methods with the 
capabilities to create three dimensional spectra of electronic, vibrational, and vibronic states and to use these spectra to determine excited state structure.

\subsection{Doubly Vibrationally Enhanced (DOVE) Four Wave Mixing (FWM) Spectroscopy}

The three Doubly Vibrationally Enhanced (DOVE) pathways in Fig. 8 consist of two vibrational transitions and a Raman transition. ${ }^{15,16,53,64,82-86}$ One of these three transitions must involve a combination band. The $v+v$ ' combination band is the common choice. The first two interactions in the DOVE-Raman process can be viewed as a vibrationally resonant CARS process. The output coherence of the DOVE-Raman pathway has the opposite phase from the output coherence of the DOVE-IR2 pathway. The two pathways will cancel if mechanical anharmonicity does not shift the transition frequencies. The DOVE-IR1 pathway is unusual because it does not require mechanical anharmonicity and does not necessarily vanish. ${ }^{51,54,55}$ It requires electrical anharmonicity where the transition dipole moment changes because another state was excited. The pathway becomes important for observing cross-peaks between two interacting molecular systems. Intermolecular interactions occur from dipole-dipole coupling that can then create an electrical anharmonicity. Since dipole-dipole coupling has a well-defined dependence on the angles and distance between the dipoles, electrical anharmonicities can form the basis for structural characterization of intermolecular interactions. This sensitivity to electrical anharmonicity makes DOVE-IR1 sensitive to the intermolecular interactions that dominate the mechanisms controlling life. ${ }^{54}$

Figure 11 shows three 2D DOVE spectra. ${ }^{51}$ The DOVE pathways are sketched on each spectrum. The abscissa and ordinate display the relevant frequencies for the fundamental and overtone/combination band, respectively. The first uses a mixture of benzonitrile and phenyl-acetylene as a model system to create cross-peaks between the two species that reflect the intermolecular coupling. The first spectrum uses the DOVE-IR1 pathway with parallel polarized excitation beams. Phenyl-acetylene has a cross-peak from the fundamental $\mathrm{C} \equiv \mathrm{C}$ stretching mode at 2110 $\mathrm{cm}^{-1}$ and its overtone near $4225 \mathrm{~cm}^{-1}$. Benzonitrile has a cross-peak from the $\mathrm{C} \equiv \mathrm{N}$ stretching mode at $2230 \mathrm{~cm}^{-1}$ and its overtone near $4458 \mathrm{~cm}^{-1}$. The mixture has two new cross-peaks created by the intermolecular

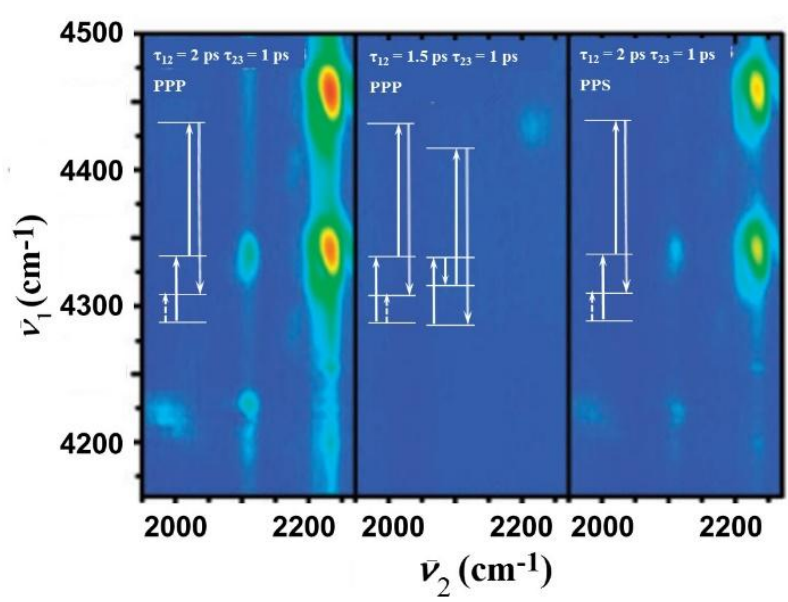

Figure 11-2D DOVE-IR spectra of a 1:1 mixture of benzonitrile and phenylamine for the time orderings, time delays, and polarization conditions indicated in the figure. The $x$ - and $y$ axes are the frequencies of the lowest and highest IR excitation frequencies. The $x$ - and $y$-axes are the frequencies of the lowest and highest IR excitation frequencies. The inserts show the coherence pathways for each time ordering. interactions where each excitation beam excites a transition on different species. The $\left(\bar{v}_{1}, \bar{v}_{2}\right)=(4335,2110) \mathrm{cm}^{-1}$ cross-peak excites the phenylacetylene first and the benzonitrile overtone mode second. The Raman output is a benzonitrile fundamental mode transition that leaves the system with a phenyl-acetylene fundamental mode 
vibration. The $\left(\bar{v}_{1}, \bar{v}_{2}\right)=(4335,2110) \mathrm{cm}^{-1}$ cross-peak excites the benzonitrile first and the phenyl-acetylene overtone mode second.

The second spectrum reverses the time ordering of the first two pulses so the DOVE-IR2 and DOVE-Raman pathways define spectrum. They destructively interfere and only the benzonitrile feature appears in the upper right. The other cross-peaks don't appear because the mechanical anharmonicity is small. The spectrum shows the superiority of the DOVE-IR1 pathway in creating cross-peaks involving intermolecular interactions.

The third spectrum is the same as the first but the third excitation beam is perpendicularly polarized. The relative intensities change in ways that reflect the differences in the angles between the transition moments in the phenyl-acetylene/benzonitrile complex and the absolute intensities reflect the distances. Comparison of the spectra with ab initio calculations of the 2D spectra then defined the distance and angle between the $\mathrm{C} \equiv \mathrm{C}$ and $\mathrm{C} \equiv \mathrm{N}$ groups in the complex. The demonstration that DOVE methods can define molecular structure in intermolecular complexes has important biological applications that are being pursued by the Klug group. ${ }^{54}$

\subsection{Triply Resonant Sum Frequency (TRSF) Spectroscopy}

Triply resonant sum frequency (TRSF) involves three sequential interactions that create an output at the sum of the three excitation frequencies. ${ }^{59,60,75}$ The discovery of TRSF was accidental and surprising. It was the result of investigating an unknown peak in a 2D DOVE spectrum. It was a peak that became the most intense feature in the spectrum when its nature was realized and the beam alignment was optimized. The normal dispersion of light should prevent phase matching TRSF because the wavelength of the nonlinear polarization is longer than the wavelength of the light it creates. There is therefore no phase matching angle that can match the wave vectors. Nevertheless, the sample path lengths used in CMDS are short enough that the perfect phase matching is not necessary and the method is an excellent complement to the DOVE pathways.

The Triply Resonant Sum Frequency (TRSF) pathway is unique and therefore independent of interference from other pathways. TRSF is a combination of two sequential and allowed infrared absorption transitions that together create an overtone or combination band. The output transition is a formally forbidden Raman combination band involving the two excited vibrational modes. The strength of this transition depends upon electronic states that are coupled to the vibrational modes. The coupling depends on FrankCondon and Hertzberg-Teller coupling that results from differences in the relative offsets and shape of the excited and ground state potential energy surfaces. Fig. 12 shows the 2D TRSF spectrum of a $300 \mu \mathrm{M}$ concentration of styryl 9B (LDS813, a laser dye) where the first two interactions occur simultaneously and the third interaction is delayed by 2.5 ps. ${ }^{60}$

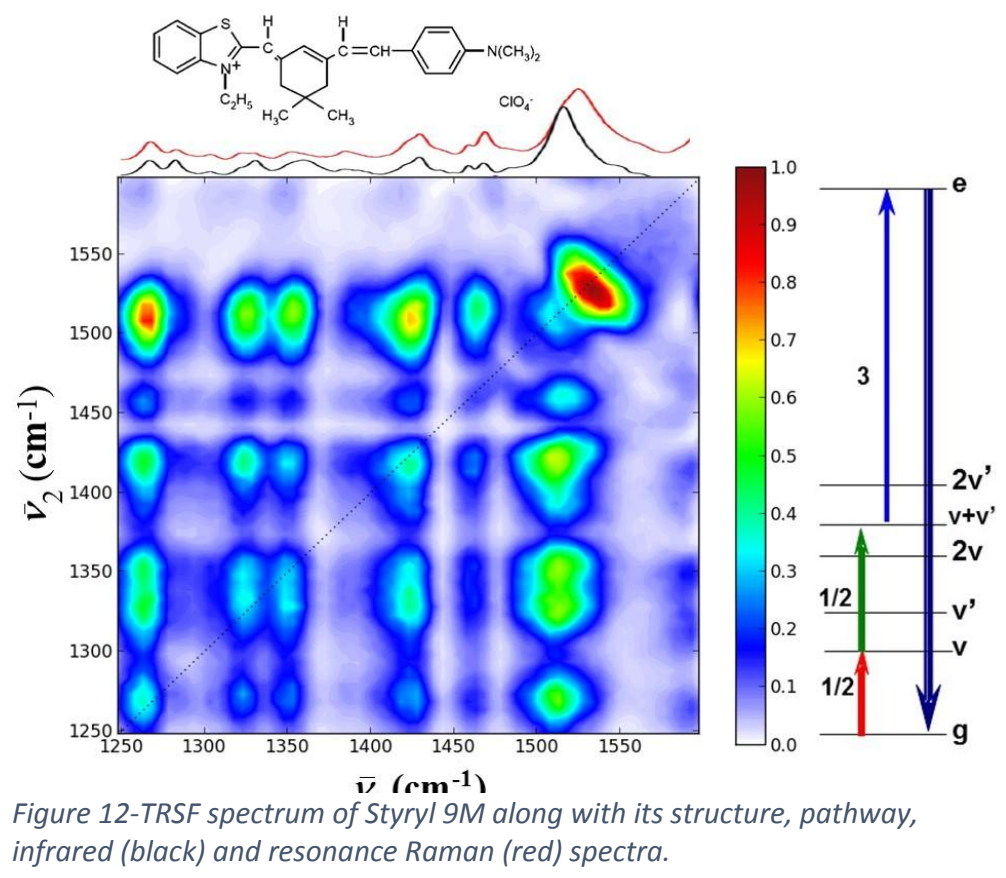


The dye does not have any symmetry and the vibrational transitions are both Raman and IR allowed. The infrared absorption (black) and resonance Raman (red) spectra (top spectra) serve as comparison references. Diagonal and cross peaks features are observed at all of the frequencies present in the infrared and Raman spectra.

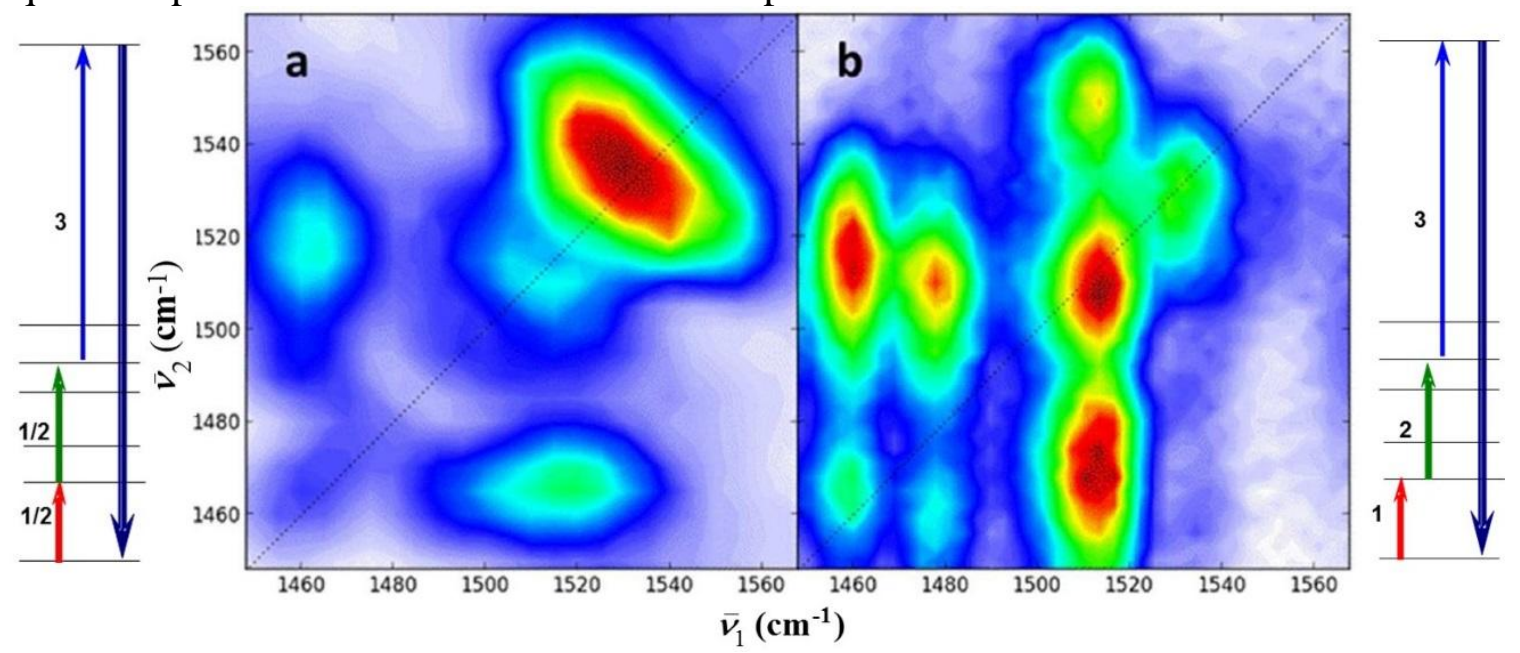

Figure 13-Expanded TRSF spectra of Figure 12. The left side shows the spectrum when the first two interactions are temporally overlapped. The right side shows the spectrum when there is a $1.5 \mathrm{ps}$ delay between the first two interactions.

The temporal overlap of the first two interactions does not distinguish between which excitation beam causes the first transition. Figure 13 shows the spectral changes when a $1.75 \mathrm{ps}$ delay is inserted between the first two excitation pulses. ${ }^{60}$ The first interaction involving $\bar{v}_{1}$ excites a fundamental mode and the second interaction involving $\bar{v}_{2}$ excites a combination band or overtone. The $\bar{v}_{2}$ frequency of a overtone or combination band is shifted from the frequency of the fundamental transition by the anharmonicity. The spectra also resolve the antidiagonal feature at $\left(\bar{v}_{1}, \bar{v}_{2}\right)=(1530,1530) \mathrm{cm}^{-1}$ in Fig. 13 into peaks located at $(1512,1545)$ and $(1531,1527) \mathrm{cm}^{-1}$. These peaks are attributed to a Fermi resonance with a state at $3057 \mathrm{~cm}^{-1}$. The antidiagonal character is expected since the Fermi resonance enhancement of the second coherence depends upon $\bar{v}_{1}+\bar{v}_{2}$. Also note that the peaks at $\bar{v}_{1}=1460 \mathrm{~cm}^{-1}$ split into two peaks. This splitting is the result of quantum beating. Quantum beating is usually understood as the temporal beating between two coherences but in the frequency domain, the beating appears as a spectral splitting with a period corresponding to the difference between the two coherence frequencies. $^{87}$

Note that several features have frequency shifts that are smaller than their homogeneous widths and therefore they have small mechanical anharmonicities. Since the TRSF pathway is unique, it is not sensitive to the mechanical anharmonicities that are required in partially coherent pathways. Like the DOVE-IR1 pathway, these features appear because of electrical anharmonicity and therefore are sensitive to intermolecular interactions. ${ }^{51}$ This sensitivity to intermolecular interactions makes DOVE-IR1 and TRSF important pathways for biological applications. $^{54,55}$

\section{Higher Order CMDS}


Multidimensional NMR routinely uses many pulses to create and manipulate multiple quantum coherences. The CMDS analogue is increasing the number of interactions with the excitation fields. Higher intensities can increase the number of interactions with a specific excitation beam. ${ }^{88-91}$ For example, the higher order processes occurring at high intensities in a three beam, four wave mixing experiment will have the phase matching condition $\vec{k}_{\text {out }}= \pm n_{1} \vec{k}_{1} \pm n_{2} \vec{k}_{2} \pm n_{3} \vec{k}_{3}$ so the phase matching condition can help define the number of interactions but it cannot isolate a specific order. ${ }^{92-94}$ For example, three interactions with wave vectors $\vec{k}_{1}-\vec{k}_{1}+\vec{k}_{1}$ will be indistinguishable from just one interaction, $\vec{k}_{1}$. Increasing the number of beams provides an alternative way to increase the number of interactions. A higher order wave mixing would then use a phase matching condition of $\vec{k}_{\text {out }}=\sum_{i} \pm n_{i} \vec{k}_{i}$. The feasibility for higher order, resonant CMDS depends upon the excitation intensity creating a Rabi frequency that is comparable to the coherence dephasing time. Otherwise, the quantum mechanical phase information in the MQC is lost before all the states can be excited. The highest excitation intensity is limited by dielectric breakdown, i.e. "zapping" a sample where the highest order interactions have become important. 95

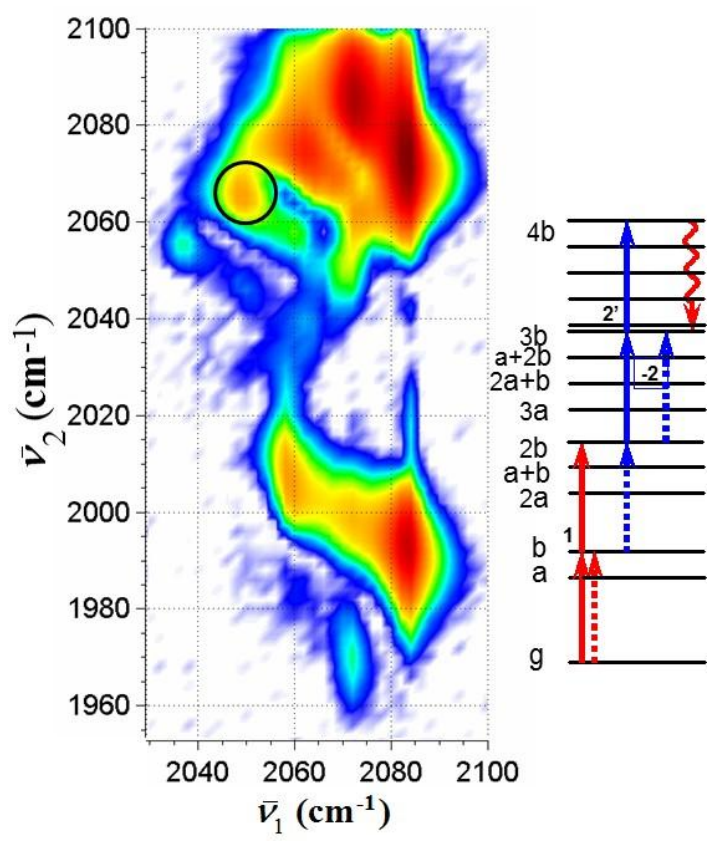

Figure 14-2D spectrum varying $\omega 2$ and $\omega m$ with $\omega 1=$ $2078 \mathrm{~cm}-1, \tau 21=1.5 \mathrm{ps}$, and $\tau 2^{\prime} 1=0$ ps. The example WMEL diagram corresponds to peak 3.

Figure 14 shows an example of a higher order CMDS method that is able to define the multidimensional potential energy surface. ${ }^{94}$ The sample is rhodium(I) dicarbonyl acetylacetonate (RDC) in a $125 \mu \mathrm{m}$ path length cell. It has asymmetric (mode a) and symmetric (mode b) CO stretch modes with stretch frequencies of $2015 \mathrm{~cm}^{-1}$ and $2084 \mathrm{~cm}^{-1}$, respectively. At low excitation intensities, the spectrum has two diagonal and two cross-peaks along with their overtone and combination band transitions. Fig. 14 resulted from measuring the output signal intensity at high excitation intensities and as a function of the $\omega_{1}$ and the $\omega_{m}$ monochromator frequencies. The color bar is logarithmic. The $\omega_{2}$ frequency was set at $2078 \mathrm{~cm}^{-1}$ in order to optimize the ladder climbing for the $\mathrm{b}$ mode. The phase matching is $\vec{k}_{\text {out }}=\vec{k}_{1}-\vec{k}_{2}+\vec{k}_{2}$. Many more features appear in the spectrum because multiple interactions occurred with each excitation beam so the series of transitions climbed the vibrational ladder in both the modes labeled $a$ and $b$. The number of interactions with each field is constrained by the phase matching condition. Nineteen features appear in the spectra corresponding to ma+nb overtones and combination bands where $m$ varies from $0-3$ and $n$ varies from $0-6$. An example WMEL diagram for the circled feature appears as an insert. Each $\omega_{m}$ frequency defines the anharmonicity of each overtone or combination band frequency. The measured anharmonicities could be fit with two anharmonic and coupled Morse potentials for the two mode modes with an average standard deviation of $1.7 \mathrm{~cm}^{-1} .96$ 
A second example of a higher order CMDS method is Resonant-Pump-Third Order Raman Spectroscopy (RaPTORS) where two field interactions excite a population in an organic probe molecule followed by three nonresonant field interactions that excite Raman modes of a solvent. ${ }^{97}$ RaPTORS is sensitive to the changes occurring in the solvent structure and the solvation dynamics following the change in the excited state geometry of the probe molecule. The nonresonant and instantaneous nature of the third order Raman probe insures that the solvation dynamics are isolated from any population dynamics occurring during the Raman probe step so the dynamics can be mapped by changing the delay between the pump excitation and the subsequent Raman probe. RaPTORS

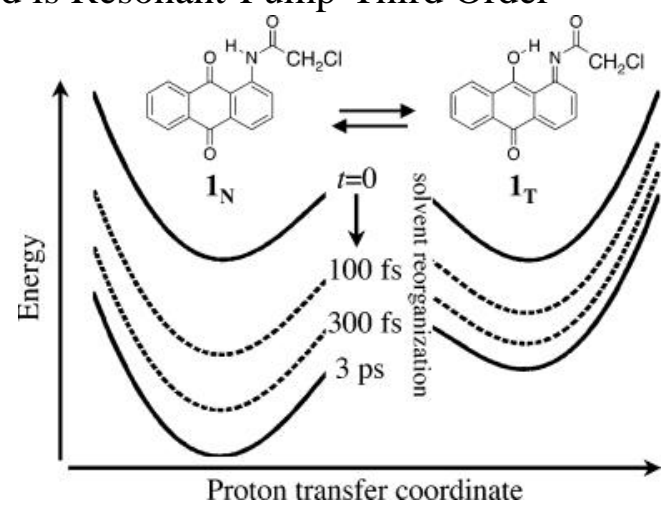
Figure 15-Schematic diagram of the time dependent changes in the potential energy surface in the intramolecular proton transfer. successfully measured the changing solvent structure created by the excitation of 1-chloroacetylaminoanthraquinone (CAAQ). Figure 15 illustrates how the changes in solvent structure affect the potential energy surface and enable excited state intramolecular proton transfer, ESIPT, where the enamine transfers a proton to the ketone converting it to an alcohol and leaving an imine. ${ }^{98}$

\subsection{Multidimensional Sum Frequency Generation (SFG) and Interface Selective Methods}

The previous CMDS examples all involved odd order nonlinear susceptibilities or even order wave mixing (e.g. FWM). Even order nonlinear susceptibilities vanish in isotropic materials because the nonlinear polarization reverses exactly between the positive and negative excursions of the electromagnetic excitation fields. They do not vanish at interfaces that break the symmetry so the positive and negative excursions no longer cancel. They therefore isolate asymmetries like interfaces. Sum frequency generation (SFG) is a very important example. ${ }^{18,19}$ SFG is a fully coherent method where two fields interact to create an output field. It is phase matched by the condition $\vec{k}_{\text {out }}=\vec{k}_{1}+\vec{k}_{2}$ where the first field usually excites a vibrational transition and the second excites a vibrational or an electronic state, either resonantly or nonresonantly. Two dimensional SFG requires a higher order five wave mixing process involving $\chi^{(4)} \cdot{ }^{99-104}$ Usually, the higher order process involves a pump-SFG probe. ${ }^{99,105,106}$ The method can be implemented as either a frequency or time domain spectroscopy.

\section{Multidimensional Spectral Signatures and the Future of CMDS}

The creation of MQCs by the different CMDS methods described above demonstrate many examples of multidimensional spectral signatures and the higher selectivity that they bring to chemical measurement. These CMDS examples primarily used simple model systems and partially coherent pathways that provide both the selectivity and the measurement of ultrafast dynamics. They demonstrate the feasibility for measuring fundamental processes that cannot be measured by other approaches. Their selectivity is currently limited because the probe step is one dimensional.

Most analytical spectroscopic methods target the very wide range of much more complex samples and materials from a broad ranges of scientific fields, samples and materials that are 
important for technology and consumer applications. The selectivity available with traditional spectroscopic methods limits their measurement capabilities in analyzing and probing complex samples and it is here that CMDS will make its most important contribution. Most incoherent spectroscopic applications use spectroscopic signatures to define changes in samples on time scales varying from ultrafast to multiyear time scales. The optimal way to use CMDS methods for the same purpose is to separate the measurement of the dynamics from the selectivity of a probe using a pump-CMDS probe method. The pump initiates the population dynamics while the CMDS probe is multidimensional and provides the high selectivity required for complex samples. Pump-CMDS probe is a higher order CMDS method. RaTORS is an example of a six wave mixing process where the nonresonant Raman probe provides an instantaneous and definitive probe that lacks its own population dynamics. It also lacks the resonant features that define a spectroscopic signature but it is still a fully coherent probe that confines the dynamics measurement to the pump-probe delay. 2D-SFG is a second example where the pump creates a vibrational or electronic population and the probe is a fully coherent SFG process that provides a 1D spectral signature.

In pump-CMDS probe spectroscopy, the pump step can either be an explicit photon excitation or any other kind of perturbation that changes the sample. In this sense, measuring the aging of a material can be considered the pump step. The pump step can also involve creating a spatial modulation of the sample properties. The spatial modulation becomes part of the phase matching involved in the CMDS probe step and defines the directionality of the output beam through the phase matching, $\vec{k}_{\text {out }}=\vec{k}_{\text {pump }}-\sum_{i=C M D S} \pm \vec{k}_{i}$. If a grating is formed by crossed pump beams, $\vec{k}_{\text {pump }}=\vec{k}_{p 1}-\vec{k}_{p 2}$. Transient grating experiments create gratings by crossing two excitation beams but they can also be created by other methods such as lithography.

Spectral signatures should ideally provide unique and invariant fingerprints. Fully coherent CMDS pathways are ideal for the probe step because they have well-defined multidimensional spectral signatures and do not involve intermediate populations that have their own dynamics such as population transfer, quenching, and spectral diffusion. ${ }^{17}$ Fully resonant four wave mixing CMDS provides three resonance enhancements that serve as multidimensional spectral signatures. The resonances can include vibrational transitions that provide high selectivity and electronic transitions that provide large resonance enhancements. The output frequencies are resolved from the excitation frequencies so spectral discrimination can remove scattering interference that often limits partially coherent pathways such as those in pump-probe, transient absorption, transient grating, and photon echo pathways. Frequency domain CMDS does not require the stringent phase stability conditions required for time domain CMDS. The phase relationships between excitation beams are only required during the creation of the multiple quantum coherences. Even more importantly, the freedom from long term phase coherence allows any combination of multiply colored excitation pulses. That makes the entire range of vibrational and electronic transitions available. This wide range is crucial if CMDS is to become a dominant technology in science.

The future of CMDS depends on two important directions:

1) Demonstration of how the CMDS methodology can tackle important problems that cannot be solved by traditional methods. CMDS as a field is rapidly expanding into many different types of applications. However, the field is still dominated by applications involving physical chemistry. It is very important to broaden the base of scientists involved in the 
development of this amazing new field if it is reach its full potential to provide advanced selectivity to all fields of science.

2) Continued development of the CMDS technology to lower the cost, the complexity, and the expertise required for using the methodology. There have been many innovations in the technology required for CMDS such as Gradient Assisted Photon Echo Spectroscopy (GRAPES $^{40,107}$ that can acquire a $2 \mathrm{D}$ spectrum on a single shot and pulse shaping ${ }^{108-112}$ that can create phase stable excitation pulses. Nevertheless, CMDS remains a daunting methodology that hinders the dissemination of the technology outside the field of nonlinear spectroscopy. CMDS would benefit from commercialization of technology that focused on integrating all aspects of CMDS into a compact and easily used system. In addition, it is very important to develop practical coherent sources that can create multiple excitation beams that are tunable over the wide range of frequencies that characterize modern spectroscopy.

\section{Acknowledgements}

The author is incredibly grateful to the 44 students who brought this field from a simple idea in 1978 to its current stage today. I am particularly grateful to Roger J. Carlson. Roger was an incredibly gifted and dedicated graduate student who developed both the theoretical and experimental foundations for CMDS in a two volume thesis that has served as the Bible over all the years for our research group. I am also incredibly grateful for the support of the Chemistry Division of the National Science Foundation over all the years of this project, most recently through grant CHE-1410510.

\section{Figure Captions}

Figure 1- Three example coherence pathways. The last arrow is the output created by the $c a$ coherence. The solid and dotted arrows specify the transitions responsible for reaching the states in the output coherence. Time evolves from left to right. The first two pathways are fully coherent and differ only in the time ordering of the transitions. The last pathway is partially coherent because the first two interactions create an a population. The bottom cartoon shows three excitation pulses and the oscillating coherences resulting after each transition.

Figure 2- 2D-IR spectra of an amyloid peptide at different times in the formation of amyloid fibers. Adapted with permission from reference 34. Copyright (2008) American Chemical Society.

Figure 3-2D-IR spectra of a vibrational probe molecule in a self-assembled monolayer on gold for two different population times. The dashed lines define the diagonal. Reprinted with permission from reference 35. Copyright (2006) National Academy of Sciences, USA

Figure 4- 2D-IR spectra of a phenol-benzene mixture for $200 \mathrm{fs}$ and 14 ps population delay times. The free phenol vibrational mode appears at $2665 \mathrm{~cm}^{-1}$. It shifts to $2631 \mathrm{~cm}^{-1}$ when the 
phenol forms a complex with benzene. Reprinted with permission from reference 36. Copyright (2006) American Chemical Society.

Figure 5-2D-IR spectra of an octapeptide using pathways with the phase matching conditions $-\vec{k}_{1}+\vec{k}_{2}+\vec{k}_{3}, \vec{k}_{1}-\vec{k}_{2}+\vec{k}_{3}, \vec{k}_{1}+\vec{k}_{2}-\vec{k}_{3}$, respectively, and two different sets of polarizations. Adapted with permission from reference 39. Copyright (2006) American Chemical Society.

Figure 6- 2D-IR spectrum of methylglycolate with each region of peaks normalized individually. The numbers denote the relative magnifications. The side plots show the absorption spectra. Reprinted with permission from reference 43. Copyright (2014) American Institute of Physics.

Figure 7- The GSB, SE, and ESA pathways for the photon pathways of a vibrational state, $a$, and its overtone state, $2 a$. The boxes denote the initial $g g$ ground state population and the $a g$ and $2 a, a$ output coherences.

Figure 8- Fully coherent pathways involving v and v' fundamental, overtone, and combination band states and e, e+v, and $e+v$ ' excited electronic and vibronic states. The interactions leading to the states involving in the output coherences appear as solid and dotted arrows.

Figure 9- Absorption (top spectrum) and 4 representative fully resonant CARS spectra of pentacene in a benzoic acid crystal at $2 \mathrm{~K}$. The first excitation is resonant with the $\mathrm{S}_{1}$ electronic transition. The second transition is resonant with the indicated vibrational mode. The third transition is scanned across the different vibronic modes. The position where $\omega_{1}-\omega_{3}$ is indicated by a D. Adapted with permission from reference 81. Copyright (1991) American Chemical Society.

Figure 10- Correlation map between the frequencies of the vibronic states in the absorption spectrum (top) and the vibrational states in the fluorescence spectra (bottom). Reprinted with permission from reference 68. Copyright (1990) American Chemical Society.

Figure 11-2D DOVE-IR spectra of a 1:1 mixture of benzonitrile and phenylamine for the time orderings, time delays, and polarization conditions indicated in the figure. The $\mathrm{x}$ - and $\mathrm{y}$ - axes are the frequencies of the lowest and highest IR excitation frequencies. The inserts show the coherence pathways for each time ordering. Reprinted with permission from reference 51 . Copyright (2006) Royal Society of Chemistry.

Figure 12- TRSF spectrum of Styryl 9M along with its structure, pathway, infrared (black) and resonance Raman (red). Adapted with permission from reference 60. Copyright (2013) American Chemical Society.

Figure 13- Expanded TRSF spectra of Figure 12. The left side shows the spectrum when the first two interactions are temporally overlapped. The right side shows the spectrum when there is a 1.5 ps delay between the first two interactions. Adapted with permission from reference 60 . Copyright (2013) American Chemical Society. 
Figure 14- 2D spectrum varying $\omega_{2}$ and $\omega_{\mathrm{m}}$ with $\omega_{1}=2078 \mathrm{~cm}^{-1}, \tau_{21}=1.5 \mathrm{ps}$, and $\tau_{2^{\prime} 1}=0 \mathrm{ps}$. The example WMEL diagram corresponds to peak 3. Adapted with permission from reference 94. Copyright (2009) American Chemical Society.

Figure 15- Schematic diagram of the time dependent changes in the potential energy surface in the intramolecular proton transfer. Reprinted with permission from reference 98. Copyright (2004) American Chemical Society. 


\section{References}

(1) Mandal, P. K.; Majumdar, A. Concepts in Magnetic Resonance Part A 2004, 20A, 1.

(2) Grzesiek, S.; Ikura, M.; Clore, G. M.; Gronenborn, A. M.; Bax, A. Journal of Magnetic Resonance 1992, 96, 215.

(3) Munowitz, M.; Pines, A. Science 1986, 233, 525.

(4) Klein, A. D.; Braun, W.; Wuthrich, K. Journal of Molecular Biology 1988, 204, 675.

(5) Wright, J. C.; Chen, P. C.; Hamilton, J. P.; Zilian, A.; LaBuda, M. J. Appl Spectrosc 1997, 51,

949.

(6) Scheurer, C.; Mukamel, S. J Chem Phys 2002, 116, 6803.

(7) Pakoulev, A. V.; Rickard, M. A.; Meyers, K. A.; Kornau, K.; Mathew, N. A.; Thompson, D. C.; Wright, J. C. J Phys Chem A 2006, 110, 3352.

(8) Fournier, F.; Gardner, E. M.; Kedra, D. A.; Donaldson, P. M.; Guo, R.; Butcher, S. A.;

Gould, I. R.; Willison, K. R.; Klug, D. R. P Natl Acad Sci USA 2008, 105, 15352.

(9) Wright, J. C. In Annual Review of Physical Chemistry, Vol 62; Leone, S. R., Cremer, P. S., Groves, J. T., Johnson, M. A., Eds. 2011; Vol. 62, p 209.

(10) Wright, J. C.; Carlson, R. J.; Hurst, G. B.; Steehler, J. K.; Riebe, M. T.; Price, B. B.; Nguyen, D. C.; Lee, S. H. International Reviews of Physical Chemistry 1991, 10, 349.

(11) Cho, M. H. Chem Rev 2008, 108, 1331.

(12) Mukamel, S. Annual Reviews of Physical Chemistry 2000, 51, 691.

(13) Jonas, D. M. Annu Rev Phys Chem 2003, 54, 425.

(14) Mukamel, S. Principles of Nonlinear Optical Spectroscopy; 1st ed.; Oxford University Press: New York, 1995.

(15) Zhao, W.; Wright, J. C. Phys Rev Lett 1999, 83, 1950.

(16) Zhao, W.; Wright, J. C. Phys Rev Lett 2000, 84, 1411.

(17) Fournier, F.; Gardner, E. M.; Guo, R.; Donaldson, P. M.; Barter, L. M. C.; Palmer, D. J.;

Barnett, C. J.; Willison, K. R.; Gould, I. R.; Klug, D. R. Analytical Biochemistry 2008, 374, 358.

(18) Du, Q.; Freysz, E.; Shen, Y. R. Science 1994, 264, 826.

(19) Huang, J. Y.; Shen, Y. R. Physical Review 1994, 49, 3973.

(20) Lee, D.; Albrecht, A. C. Advances in Infrared and Raman Spectroscopy; 1st ed.; WileyHeyden: Chichester, 1985; Vol. 12.

(21) Wright, J. C. International Reviews of Physical Chemistry 2002, 21, 185.

(22) Zanni, M. T.; Asplund, M. C.; Hochstrasser, R. M. J Chem Phys 2001, 114, 4579.

(23) Hybl, J. D.; Ferro, A. A.; Jonas, D. M. J Chem Phys 2001, 115, 6606.

(24) Hybl, J. D.; Albrecht, A. W.; Faeder, S. M. G.; Jonas, D. M. Chem Phys Lett 1998, 297, 307.

(25) Gallagher, S. M.; Albrecht, A. W.; Hybl, J. D.; Landin, B. L.; Rajaram, B.; Jonas, D. M.

Journal of the Optical Society of America B 1998, 15, 2338.

(26) Chatzidimitriou-Dreismann, C. A.; Tedah, T. A.; Streffer, R. M. F.; Mayers, J. Phys Rev Lett 1997, 79, 2839.

(27) Mukherjee, P.; Kass, I.; Arkin, I.; Zanni, M. T. P Natl Acad Sci USA 2006, 103, 8571.

(28) Fuller, F. D.; Pan, J.; Gelzinis, A.; Butkus, V.; Senlik, S. S.; Wilcox, D. E.; Yocum, C. F.;

Valkunas, L.; Abramavicius, D.; Ogilvie, J. P. Nat Chem 2014, 6, 706.

(29) Rubtsov, I. V.; Wang, J. P.; Hochstrasser, R. M. P Natl Acad Sci USA 2003, 100, 5601.

(30) Aartsma, T. J.; Wiersma, D. A. Phys Rev Lett 1976, 36, 1360.

(31) Tokmakoff, A.; Fayer, M. D. Accounts of Chemical Research 1995, $28,437$.

(32) Aartsma, I. J.; Wiersma, D. A. Chem Phys Lett 1976, 42, 520.

(33) Berg, M.; Walsh, C. A.; Narasimhan, L. R.; Littau, K. A.; Fayer, M. D. J Chem Phys 1988, $88,1564$.

(34) Strasfeld, D. B.; Ling, Y. L.; Shim, S. H.; Zanni, M. T. J Am Chem Soc 2008, 130, 6698. 
(35) Yan, C.; Yuan, R.; Pfalzgraff, W. C.; Nishida, J.; Wang, L.; Markland, T. E.; Fayer, M. D. Proc Natl Acad Sci U S A 2016, 113, 4929.

(36) Kwac, K.; Lee, C.; Jung, Y.; Han, J.; Kwak, K.; Zheng, J. R.; Fayer, M. D.; Cho, M. J Chem Phys 2006, 125, 244508.

(37) Kwak, K.; Zheng, J. R.; Cang, H.; Fayer, M. D. J Phys Chem B 2006, 110, 19998.

(38) Naraharisetty, S. R. G.; Kasyanenko, V. M.; Rubtsov, I. V. J Chem Phys 2008, 128, 104502.

(39) Sul, S.; Karaiskaj, D.; Jiang, Y.; Ge, N. H. J Phys Chem B 2006, 110, 19891.

(40) Harel, E.; Fidler, A. F.; Engel, G. S. J Phys Chem A 2011, 115, 3787.

(41) Bian, H. T.; Li, J. B.; Wen, X. W.; Sun, Z. G.; Song, J. A.; Zhuang, W.; Zheng, J. R. J Phys Chem A 2011, 115, 3357.

(42) Rubtsova, N. I.; Rubtsov, I. V. Annu Rev Phys Chem 2015, 66, 717.

(43) Leger, J. D.; Nyby, C. M.; Varner, C.; Tang, J. A.; Rubtsova, N. I.; Yue, Y. K.; Kireev, V. V.;

Burtsev, V. D.; Qasim, L. N.; Rubtsov, G. I.; Rubtsov, I. V. Review of Scientific Instruments 2014, 85, 16.

(44) Tekavec, P. F.; Lott, G. A.; Marcus, A. H. J Chem Phys 2007, 127, 214307.

(45) Tekavec, P. F.; Dyke, T. R.; Marcus, A. H. J Chem Phys 2006, 125, 194303.

(46) Karki, K. J.; Widom, J. R.; Seibt, J.; Moody, I.; Lonergan, M. C.; Pullerits, T.; Marcus, A. H. Nat Commun 2014, 5, 7.

(47) Pisliakov, A. V.; Mancal, T.; Fleming, G. R. J Chem Phys 2006, 124, 234505.

(48) Cheatum, C. M.; Tokmakoff, A.; Knoester, J. J Chem Phys 2004, 120, 8201.

(49) Scheurer, C.; Piryatinski, A.; Mukamel, S. J Am Chem Soc 2001, 123, 3114.

(50) Khalil, M.; Tokmakoff, A. Chemical Physics 2001, 266, 213.

(51) Guo, R.; Fournier, F.; Donaldson, P. M.; Gardner, E. M.; Gould, I. R.; Klug, D. R. Phys Chem Chem Phys 2009, 11, 8417.

(52) Cho, M. Phys Rev A 2000, 61, 023406.

(53) Kwak, K.; Cha, S.; Cho, M. H.; Wright, J. C. J Chem Phys 2002, 117, 5675.

(54) Guo, R.; Mukamel, S.; Klug, D. R. PCCP 2012, 14, 14023.

(55) Guo, R.; Miele, M.; Gardner, E. M.; Fournier, F.; Kornau, K. M.; Gould, I. R.; Klug, D. R. Faraday Discuss. 2011, 150, 161.

(56) Golonzka, O.; Khalil, M.; Demirdoven, N.; Tokmakoff, A. Phys Rev Lett 2001, 86, 2154.

(57) Pakoulev, A. V.; Rickard, M. A.; Kornau, K. M.; Mathew, N. A.; Yurs, L. A.; Block, S. B.;

Wright, J. C. Accounts of Chemical Research 2009, 42, 1310.

(58) Kuznetsova, I.; Thomas, P.; Meier, T.; Zhang, T.; Li, X.; Mirin, R. P.; Cundiff, S. T. Solid State Commun 2007, 142, 154.

(59) Boyle, E. S.; Pakoulev, A. V.; Wright, J. C. J Phys Chem A 2013, 117, 5578.

(60) Boyle, E. S.; Neff-Mallon, N. A.; Wright, J. C. J Phys Chem A 2013, 117, 12401.

(61) Besemann, D. M.; Meyer, K. A.; Wright, J. C. J Phys Chem B 2004, 108, 10493.

(62) Meyer, K. A.; Besemann, D. M.; Wright, J. C. Chem Phys Lett 2003, 381, 642.

(63) Zhao, W.; Murdoch, K. M.; Besemann, D. M.; Condon, N. J.; Meyer, K. A.; Wright, J. C. Appl Spectrosc 2000, 54, 1000.

(64) Zhao, W.; Wright, J. C. J Am Chem Soc 1999, 121, 10994.

(65) Zilian, A.; LaBuda, M. J.; Hamilton, J. P.; Wright, J. C. J. Lumines. 1994, 60\&61, 655.

(66) Ivanecky, J. E.; Wright, J. C. Chem Phys Lett 1993, 206, 437.

(67) Carlson, R. J.; Wright, J. C. Journal of Molecular Spectroscopy 1990, 143, 1.

(68) Carlson, R. J.; Nguyen, D. C.; Wright, J. C. J Chem Phys 1990, 92, 1538.

(69) Carlson, R. J.; Wright, J. C. J Chem Phys 1990, 92, 5186.

(70) Steehler, J. K.; Wright, J. C. Chem Phys Lett 1985, 115, 486.

(71) Steehler, J. K.; Wright, J. C. J Chem Phys 1985, 83, 3188.

(72) Steehler, J. K.; Wright, J. C. J Chem Phys 1985, 83, 3200. 
(73) Nguyen, D. C.; Wright, J. C. Chem Phys Lett 1985, 117, 224.

(74) Lee, S. H.; Steehler, J. K.; Nguyen, D. C.; Wright, J. C. App/ Spectrosc 1985, 39, 243.

(75) Boyle, E. S.; Neff-Mallon, N. A.; Handali, J. D.; Wright, J. C. J Phys Chem A 2014, 118,

3112.

(76) Hurst, G. B.; Wright, J. C. J Chem Phys 1992, 97, 3940.

(77) Wright, J. C.; Carlson, R. J.; Riebe, M. T.; Steehler, J. K.; Nguyen, D. C.; Lee, S. H.; Price, B. B.; Hurst, G. B. In Vibrational Spectroscopy and Structure; 1st ed.; Bist, H. D., Durig, J. R., Sullivan, J. F., Eds.; Elsevier Science Publishers: Amsterdam, 1989; Vol. 17B, p 123.

(78) Riebe, M. T.; Wright, J. C. J Chem Phys 1988, 88, 2981.

(79) Riebe, M. T.; Wright, J. C. Chem Phys Lett 1987, 138, 565.

(80) Nguyen, D. C.; Wright, J. C. App/ Spectrosc 1985, 39, 230.

(81) Carlson, R. J.; Wright, J. C. Analytical Chemistry 1991, 63, 1449.

(82) Condon, N. J.; Wright, J. C. J Phys Chem A 2005, 109, 721.

(83) Meyer, K. A.; Wright, J. C. Analytical Chemistry 2001, 73, 5020.

(84) Meyer, K. A.; Wright, J. C. J Phys Chem A 2003, 107, 8388.

(85) Murdoch, K. M.; Condon, N. J.; Zhao, W.; Besemann, D. M.; Meyer, K. A.; Wright, J. C. Chem Phys Lett 2001, 335, 349.

(86) Wright, J. C.; Condon, N. J.; Murdoch, K. M.; Besemann, D. M.; Meyer, K. A. J Phys Chem A 2003, 107, 8166 .

(87) Pakoulev, A. V.; Rickard, M. A.; Mathew, N. A.; Kornau, K. M.; Wright, J. C. J Phys Chem A 2007, 111, 6999.

(88) Winker, B. K.; Wright, J. C. Opt Lett 1988, 13, 913.

(89) Winker, B. K.; Wright, J. C. Analytical Chemistry 1988, 60, 2599.

(90) Winker, B. K.; Wright, J. C. Opt Lett 1988, 13, 913.

(91) Winker, B. K.; Wright, J. C. Opt Lett 1989, 14, 54.

(92) Mathew, N. A.; Block, S. B.; Yurs, L. A.; Kornau, K. M.; Pakoulev, A. V.; Wright, J. C. J Phys Chem A 2009, 113, 13562.

(93) Mathew, N. A.; Yurs, L. A.; Block, S. B.; Pakoulev, A. V.; Kornau, K. M.; Sibert, E. L.; Wright, J. C. J Phys Chem A 2010, 114, 817.

(94) Mathew, N. A.; Yurs, L. A.; Block, S. B.; Pakoulev, A. V.; Kornau, K. M.; Wright, J. C. J Phys Chem A 2009, 113, 9261.

(95) Wright, J. C.; Wirth, M. J. Analytical Chemistry 1980, 52, A988.

(96) Mathew, N. A.; Yurs, L. A.; Block, S. B.; Pakoulev, A. V.; Kornau, K. M.; Wright, J. C. J Phys Chem A 2009, 114, 817.

(97) Underwood, D. F.; Blank, D. A. J Phys Chem A 2003, 107, 956.

(98) Schmidtke, S. J.; Underwood, D. F.; Blank, D. A. J Am Chem Soc 2004, 126, 8620.

(99) Yamaguchi, S.; Tahara, T. J Chem Phys 2006, 125, 194711.

(100) Singh, P. C.; Nihonyanagi, S.; Yamaguchi, S.; Tahara, T. J Chem Phys 2013, 139, 161101.

(101) Singh, P. C.; Nihonyanagi, S.; Yamaguchi, S.; Tahara, T. J Chem Phys 2012, 137, 094706.

(102) Xiong, W.; Laaser, J. E.; Mehlenbacher, R. D.; Zanni, M. T. Proc Natl Acad Sci U S A 2011, 108, 20902.

(103) Bredenbeck, J.; Ghosh, A.; Smits, M.; Bonn, M. J Am Chem Soc 2008, 130, 2152.

(104) Zhang, Z.; Piatkowski, L.; Bakker, H. J.; Bonn, M. Nat Chem 2011, 3, 888. $86,1566$.

(105) Bonn, M.; Hess, C.; Miners, J. H.; Heinz, T. F.; Bakker, H. J.; Cho, M. Phys Rev Lett 2001,

(106) Tahara, T. In International Conference on Coherent Multidimensional Spectroscopy Kyoto, Japan, 2008.

(107) Harel, E.; Fidler, A. F.; Engel, G. S. P Natl Acad Sci USA 2010, 107, 16444. 
(108) Strasfeld, D. B.; Shim, S. H.; Zanni, M. T. In Advances in Chemical Physics, Vol 141; Rice, S. A., Ed.; John Wiley \& Sons Inc: New York, 2009; Vol. 141, p 1.

(109) Strasfeld, D. B.; Shim, S. H.; Zanni, M. T. Phys Rev Lett 2007, 99.

(110) Shim, S. H.; Zanni, M. T. PCCP 2009, 11, 748.

(111) Shim, S. H.; Strasfeld, D. B.; Ling, Y. L.; Zanni, M. T. Proc. Natl. Acad. Sci. U.S.A. 2007, 104, 14197.

(112) Shim, S. H.; Strasfeld, D. B.; Fulmer, E. C.; Zanni, M. T. Opt. Lett. 2006, 31, 838. 